\title{
Cervical adenocarcinoma and adenomatoid tumor of the uterus; A rare coexisting finding and review of the literature*
}

\author{
Volkan Ulker ${ }^{1}$, Alpaslan Akyol $^{2}$, Ali Gedikbasi ${ }^{2 \#}$, Ceyhun Numanoglu ${ }^{1}$, Verda Alpay ${ }^{2}$, Can Aksu ${ }^{3}$ \\ ${ }^{1}$ Department of Obstetrics and Gynecology, Oncology Unit, Kanuni Sultan Suleyman Teaching and Research Hospital, Istanbul, \\ Turkey \\ ${ }^{2}$ Department of Obstetrics and Gynecology, Kanuni Sultan Suleyman Teaching and Research Hospital, Istanbul, Turkey \\ ${ }^{3}$ Department of Pathology, Istanbul Faculty of Medicine, Istanbul University, Istanbul, Turkey \\ Email: ${ }^{*}$ alged 1971@yahoo.com
}

Received 20 September 2012; revised 22 October 2012; accepted 31 October 2012

\begin{abstract}
Genital origin adenocarcinomas with coexisting uterine adenomatoid tumors are very rare with only four published cases in the literature. We report a case of adenomatoid tumor of the uterus that was found incidentally during treatment of cervical adenocarcinoma. Although adenomatoid tumors may cause a differential diagnostic problem, in our case the hematoxylin-eosin histological and immunhistochemical studies confirmed mesothelial phenotype of tumor and these also helped in differentiating an adenomatoid tumor from the carcinomatous myometrial invasion. Adenomatoid tumors are easily recognizable benign pathologies. An incidental finding of adenomatoid tumors accompanied by adenocarcinoma of the endometrium or cervix should always be considered.
\end{abstract}

Keywords: Adenomatoid Tumor; Cervical Adenocarcinoma

\section{INTRODUCTION}

Adenomatoid tumors are uncommon, benign neoplasms of mesothelial origin, occurring most frequently in the uterus, fallopian tube and ovarian hilus in females [1]. Patients with these tumors are commonly asymptomatic and tend to mimic uterine fibroids clinically and radiologically [2]. Therefore they are almost always incidental findings at postoperative histological examination. Adenomatoid tumors may be accompanied by adenocarcinoma of the endometrium or cervix and differential diagnosis must definitely be performed in this condition, because of the typical pseudoglanduler structures these

\footnotetext{
${ }^{*}$ The authors report no declarations of interest.

${ }^{\#}$ Corresponding author.
}

tumors possess. We herein report an adenomatoid tumor showing evident adenoid pattern coexisting with adenocarcinoma of uterine cervix.

\section{CASE PRESENTATION}

A 46-year-old, gravida 2, para 2 woman was referred to our gynecologic oncology department with complaints of menorrhagia and an abnormal cervical cytology report showing high grade squamous intraepithelial neoplasia (HSIL). Sonographic examination suggested two uterine fibroids with measuring $3 \times 3 \mathrm{~cm}$ intramural and $3 \times 2$ $\mathrm{cm}$ submucosal. Colposcopy-directed biopsies of the cervix, endocervical curettage (ECC), and endometrial sampling were performed. Histopathologic examination of biopsy specimens revealed mild dysplasia, noninvasive cervical adenocarcinoma and proliferative endometrium respectively. Conization with ECC was performed. Histopathology of conization specimen similarly adjusted with cervical adenocarcinoma in situ and surgical margins were tumor free. Following this hysterectomy was recommended and total abdominal hysterectomy was performed.

Gross examination revealed one uterine fibroid and one non-encapsuled, nodular mass with tan-grey cut surfaces located in deep myometrium of the low uterine segment. Microscopically, the mass was composed of numerous gland-like tubular or cystic branching spaces, lined by eosinophilic single flattened cells and these spaces were situated between fascicles of smooth muscle cells (Figure 1(A)). The numbers of mitotic figures were found $0-1$ per 10 high-power fields. Immunhistochemical staining was positive for calretinin (Figure 1(B)), negative for $\mathrm{p} 53$ and Ki-67 expression was weak and present in only $5 \%$ of cells. The histopathology of the tumor demonstrated a benign uterine adenomatoid tumor. Additionally, surgical margins of the remaining endo- 


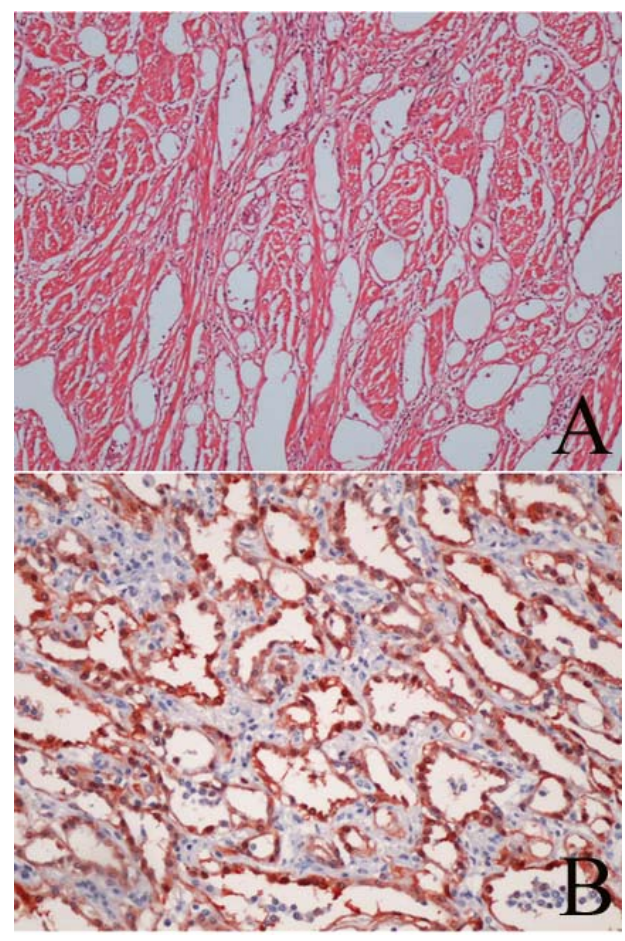

Figure 1. Numerous pseudoglanduler structures with spaces lined by epithelioid, flattened cells separated by thin septa (A) $(\mathrm{H} \& \mathrm{E} / \times 10$ high power) and immunohistochemical staining for calretinin $(\times 20$ high power $)(B)$.

cervical canal of the hysterectomy specimen after conization were tumor free. The patient had an uneventful recovery and no recurrence was detected for 18 months in the follow-up period.

\section{DISCUSSION}

Adenomatoid tumors were named after Golden and Ash in 1945 [3]. The reported relative incidence is approximately $1 \%$ in hysterectomy specimens [4] and with no documentation of malignant transformation. In female genital system they are found in the fallopian tubes, uterus and ovarian hilus; however, rare extragenital adenomatoid tumors have been in various locations, including the adrenal gland, intestinal mesentery, pleura, omentum and retroperitoneum [5].

Adenomatoid tumors evidently have a mesothelial phenotype and immunoreactivity. However, the origin of adenomatoid tumors from primitive pluripotent mesenchymal cells has also been suggested [6]. Although the histogenetic origin of adenomatoid tumors is highly debated, recognition of these tumors in genital or extra genital sites is important to prevent diagnostic confusion with other neoplasms, especially metastatic adenocarcinoma. Histopathologic features of adenomatoid tumors and adenocarcinoma are well-recognized and differential diagnosis is usually not complicated [7]. But sometimes diffuse, infiltrative growth pattern, extensive coagulation necrosis and cells with small intracytoplasmic vacuoles may cause a diagnostic dilemma and this diagnostic difficulty has led to inappropriate surgery in some of the cases [8]. In our case adenocarcinoma was limited to the endocervical mucosa of conization specimen and glandlike cystic structures invading the myometrium were located $1.5 \mathrm{~cm}$ away from the surgical margin of the remaining endocervical canal at the closest point. Despite marked hypercellularity and confluent tubular spaces there was not any mitotic activity in the well circumscribed mass and background histological pattern was typical for adenomatoid tumor. This also helped in differentiating adenomatoid tumor from the carcinomatous myometrial invasion. Numerous immunhistochemical studies demonstrated that these tumor cells have a strong positivity to low molecular weight cytokeratins, calretinin and HMBE-1 [9]. As well, the panel of immunhistochemical stains included markers of cell proliferation and genomic integrity: Ki-67 and p53. The Ki-67 protein is a nuclear and nucleolar protein that is strictly associated with cell proliferation. p53 is a tumor suppressor gene product where the mutated form, being more stable than the wild-type protein, may accumulate and be immunhistochemically detectable. In the present case, tumor cells were strongly positive for calretinin, negative for $\mathrm{p} 53$ and $\mathrm{Ki}-67$ index of mesothelial cells was $<5 \%$, supporting the mesothelial origin.

To date there have been two reports of the coexistence of these two tumors [4,7]. Previous reported cases were of endometrium origin, while in the present case it was originated from endocervix (Table 1).

Four histologic patterns of the adenomatoid tumor have been classified as adenoid, angiomatoid, solid and cystic [10]. Combination of two or more patterns occurred in each tumor. The most frequent types are the adenoid and the angiomatoid. In our case; the adenoid pattern composed the major pattern while the angiomatoid pattern was a minor element. An interesting finding is that relationship between diffuse adenomatoid tumors has been observed with immunosuppressed renal transplant recipient [8].

In conclusion, adenomatoid tumors are easily recognizable and differential diagnosis from leiomyomas, metastatic malignant tumors or primary malignant vascular

Table 1. Cases of endometrial or cervical adenocarcinoma with coexisting of uterine.

\begin{tabular}{cc}
\hline Authors & Histopathology of malignant tumor \\
\hline Nogales et al. & Endometrioid type ( 2 cases $)$ \\
Clear cell (1 case) \\
Cserni et al. & Endometrioid type (1 case) \\
Present case & Cervical adenocarcinoma (1 case) \\
\hline
\end{tabular}


tumors was made. If it is not correctly interpreted adenomatoid tumors may potentially lead to more aggressive therapy than warranted.

\section{REFERENCES}

[1] Young, R.H., Silva, E.G. and Scully, R.E. (1991) Ovarian and juxtaovarian adenomatoid tumor: A report of six cases. International Journal of Gynecological Pathology, 10, 364-371. doi:10.1097/00004347-199110000-00009

[2] Kalidindi, M. and Odejinmi, F. (2010) Laparascopic excision of uterine adenomatoid tumor: Two cases and literature review. Archives of Gynecology and Obstetrics, 281, 311-315. doi:10.1007/s00404-009-1114-0

[3] Golden, A. and Ash, J.E. (1945) Adenomatoid tumors of the genital tract. American Journal of Pathology, 21, 6379 .

[4] Nogales, F.F., Isaac, M.A., Hardisson, D., Bosincu, L., Palacios, J., Ordi, J., Mendoza, E., Manzarbeitia, F., Olivera, H., O’Valle, F., Krasević, M. and Márquez, M. (2002) Adenomatoid tumors of the uterus: An analysis of 60 cases. International Journal of Gynecological Pathology, 21, 34-40. doi:10.1097/00004347-200201000-00007

[5] Isotalo, P.A., Nascimento, A.G., Trastek, V.F., Wold, L.E. and Cheville, J.C. (2003) Extragenital adenomatoid tumor of a mediastinal lymph node. Mayo Clinic Proceedings, 78, 350-354. doi:10.4065/78.3.350

[6] Mai, K.T., Yazdi, H.M., Perkins, D.G. and Isotalo, P.A. (1999) Adenomatoid tumors of the genital tract: Evidence of mesenchymal cell origin. Pathology, Research \& Practice, 195, 605-610. doi:10.1016/S0344-0338(99)80125-3

[7] Cserni, G., Kocsis, L., Pusztai, Z. and Godo, G. (2003) Endometrial adenocarcinoma with coexisting adenomatoid tumor of the uterus. Gynecologic Oncology, 90, $207-$ 210. doi:10.1016/S0090-8258(03)00225-7

[8] Cheng, C.L. and Wee, A. (2003) Diffuse uterine adenomatoid tumor in an immunosuppressed renal transplant recipient. International Journal of Gynecological Pathology, 22, 198-201. doi:10.1097/00004347-200304000-00015

[9] Irikoma, M., Takahashi, K., Kurioka, H., Miyazaki, K. and Kamei, T. (2001) Uterine adenomatoid tumors comfirmed by immunohistochemical staining. Archives of Gynecology and Obstetrics, 265, 151-154. doi: $10.1007 / \mathrm{s} 004040000156$

[10] Quigley, J.C. and Hart, W.R. (1981) Adenomatoid tumors of the uterus. American Journal of Clinical Pathology, 76, 627-635. 